\title{
An occultation event in the nucleus of the planetary nebula M 2-29
}

\author{
M. Hajduk ${ }^{1}$, A. A. Zijlstra ${ }^{2}$, and K. Gesicki ${ }^{1}$ \\ 1 Centrum Astronomii UMK, ul.Gagarina 11, 87-100 Torun, Poland \\ e-mail: [Marcin.Hajduk;Krzysztof.Gesicki]@astri.uni.torun.pl \\ 2 University of Manchester, School of Physics \& Astronomy, Oxford Street, Manchester M13 9PL, UK \\ e-mail: a.zijlstra@manchester.ac.uk
}

Received 1 July 2008 / Accepted 9 September 2008

\section{ABSTRACT}

\begin{abstract}
Aims. Eclipses and occultations of post-AGB stars provide a powerful method of exploring the near-stellar environment, including close companions and circumstellar debris disks. Only six eclipsing systems and one dust-occultation system are currently known. New cases are important for our understanding of binary evolution during the AGB mass-loss phase.

Methods. We study the post-AGB central star of the (bipolar) Galactic bulge planetary nebula M2-29. We have obtained additional HST imaging and SAAO spectroscopy of the object.

Results. The star showed a pronounced, long-lasting occultation with subsequent recovery. The event lasted almost 3 years, with a secondary minimum 9 years later. The photometric behavior of M 2-29 resembles the dust-occultation events seen in NGC 2346, and is modeled as an occultation by a circumbinary disk, where the binary period is 18 yr. Modulation during the decline shows evidence of another companion with a period of 23 days.

Conclusions. M2-29 is the first eclipsing disk system among post-AGB stars. Close binaries with periods of around 1 month, as found in M2-29, have been proposed to supply the energy needed to create the tori of bipolar planetary nebulae.
\end{abstract}

Key words. ISM: planetary nebulae: individual: PN G004.0-03.0 - stars: AGB and post-AGB - planetary nebulae: general

\section{Introduction}

The role of binary stars in the formation and evolution of planetary nebulae (PNe) is a controversial one (Zijlstra 2007). In the traditional view, the ejection of the nebula occurs through a radiation-driven superwind, at the tip of the asymptotic giant branch (AGB); however, the outflow may instead be driven via an interacting binary system, providing a source of angular momentum to the outflow (e.g. Livio \& Soker 1988). Even if not directly interacting, binary companions can trap some previously ejected material into disks, as commonly seen around cool (A-F)post-AGB stars (van Winckel 2003) with orbital periods of 100-1000 days.

Information on binary systems and disks within PNe is limited. Only about 20 compact binary systems are known, and all but one have periods in the range 0.1-5 day (de Marco 2008). Six eclipsing systems are currently known (Miszalski et al. 2008; de Marco 2008), and they provide valuable but rare information on close companions. Their periods are between 0.17 and 4.9 days.

A few PNe show evidence of trapped circumstellar material. The VLT has detected a $10 \mathrm{AU}$ disk around the central star of the Ant nebula (Chesneau et al. 2007). Infrequent obscurations by orbiting dust clouds provide evidence of a similar structure around the central star of NGC 2346 (V651 Mon) (Méndez et al. 1982). NGC 2346 is a known binary system. With a period of 16 days it is so far the only known wider system, with a period some 5 times longer than the next longest. It approaches the period range seen for the $\mathrm{A}-\mathrm{F}$ post-AGB stars with disks.

Peretto et al. (2007) suggest that NGC 6302 has an undetected binary companion with a period of about 1 month. Such a binary could have supplied the energy necessary for its

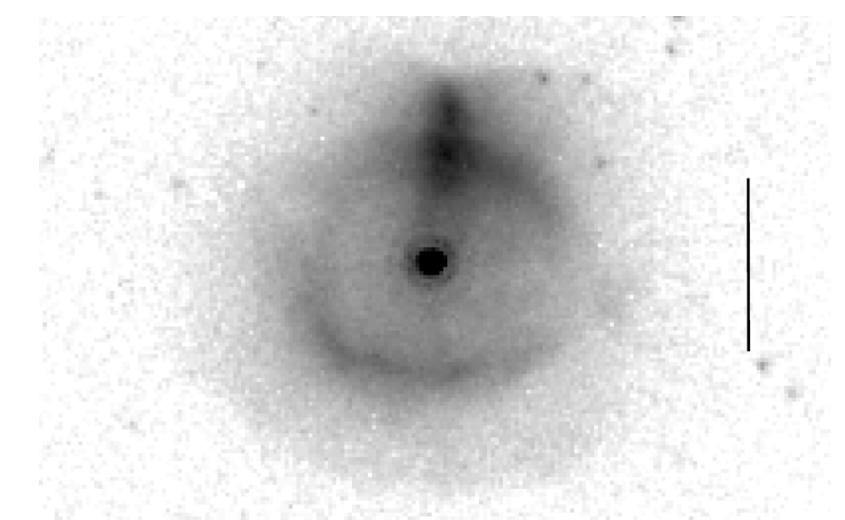

Fig. 1. H $\alpha$ HST image of M 2-29 obtained in 2003. A logarithmic intensity scale is applied. The nebula is complex, including a ring, a halo, a jet-like structure, and a central star. North is on top, east to the left. The length of the bar is 2 arcsec.

expanding, high-mass torus. If this is correct, the implication is that PNe with dense tori have binaries with similar periods and possibly central remnant disks.

The planetary nebula M2-29 (PNG004.0-03.0) is a 4-arcsec object, previously considered as a halo PN (Peña \& Torres-Peimbert 1991), but the revised abundance analysis of Torres-Peimbert et al. (1997) suggests it to be a member of the bulge. Old HST images are described by Torres-Peimbert et al. (1997). New HST Wide Field Planetary Camera 2 images in [O III] $5007 \AA$ and $\mathrm{H} \alpha$ lines and in continuum were obtained on May 20, 2003. The H $\alpha$ image (Fig. 1) confirms the features described by Torres-Peimbert et al. (1997) but at much better 


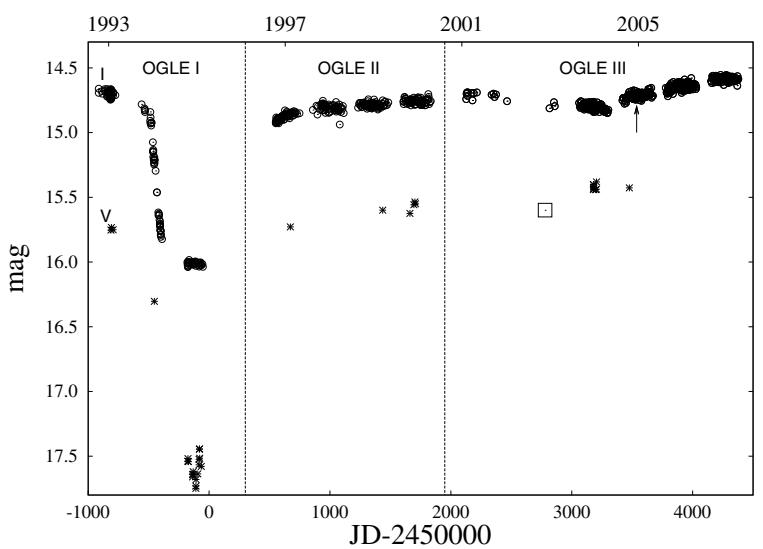

Fig. 2. $I$ and $V$ lightcurve of M 2-29 from OGLE. The rectangle gives the stellar magnitude obtained from the HST $547 \mathrm{~nm}$ image. The arrow marks the SAAO spectroscopic observation.

sensitivity. (The jet-like feature will be discussed elsewhere: we attribute it to interaction with the interstellar medium Wareing et al. 2007.) The 2MASS NIR colors of $J-H=0.41$ and $H-K=1.17$ differ from those of ionized gas, and are indicative of a hot star plus a $1000 \mathrm{~K}$ black body (Whitelock 1985).

In this paper we present data on a new occultation event in a central star of a planetary nebula, lasting several years.

\section{Observations and data reduction}

M 2-29 has been observed by the OGLE (Optical Gravitational Lensing Experiment) survey since 1992. OGLE observed the star regularly in the $I$ band and occasionally in the $V$ band. The central star of M2-29 is found to exhibit unusual light variations. Zebrun (1998) lists it as an R CrB-type star candidate. The lightcurve of the object presented in Fig. 2 shows an eclipse-like event, lasting several years (many times longer than the obscuration events in $\mathrm{RCrB}$ stars). Out of minimum, the lightcurve shows other slow changes.

The OGLE data covers three phases of the survey: OGLE-I (1993-1995), OGLE-II (1997-2000) and OGLE-III (2001 onward), over a total period of 14 years, with short periods of avoidance each year. OGLE-I operated at the 1-m Swope telescope at the Las Campanas Observatory. After a one-year break in 1996, the project moved to the dedicated 1.3-m Warsaw Telescope. OGLE-II was performed using a single SITe CCD camera, while the third phase of the project used eight binned CCD chips.

Only the OGLE epoch-II photometry is calibrated in absolute units. An average systematic shift of -0.04 and +0.26 mag at $I$ band (with an rms of 0.03) was applied for OGLE-I and OGLE-III, respectively, based on comparing the magnitudes of 8 field stars. The $I$-band pipeline photometry uses the DIA (Difference Image Analysis) method, where a reference image is subtracted before the photometry is carried out. The $V$-band pipeline photometry is done differently, using DAOPHOT PSF-fitting. To obtain correct stellar magnitudes, we measured the $V$ band magnitudes again with the DIA method, subtracting the reference image from other images.

The OGLE $V$ and $I$ filters are close to the Landolt standards (Udalski et al. 2002). The $I$ bandpass avoids strong nebular emission lines and is dominated by the stellar emission. This is confirmed by the stellar appearance of M 2-29 in the $I$ images. The $V$-image is affected by the nebular [O III] $5007 \AA$ line, located at the blue edge of the filter. The $V$ band OGLE images indeed

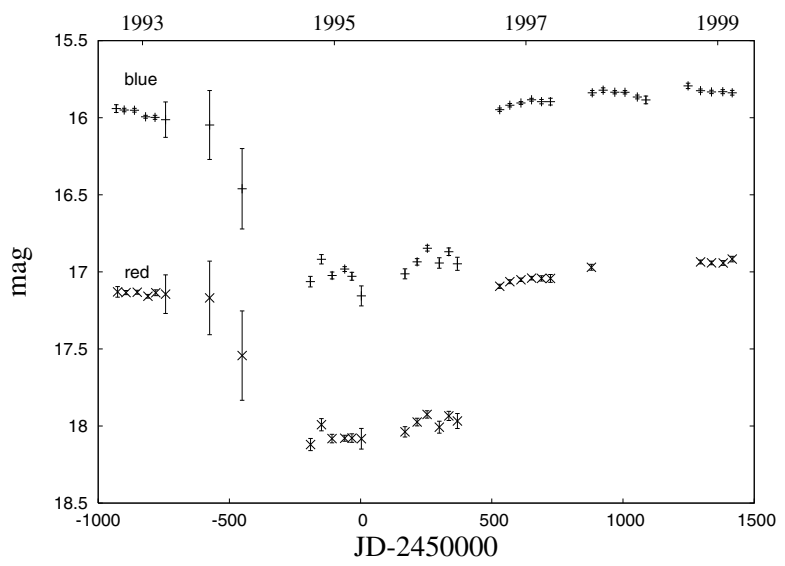

Fig. 3. Blue and red lightcurve of M 2-29 from MACHO, averaged over 40-day bins.

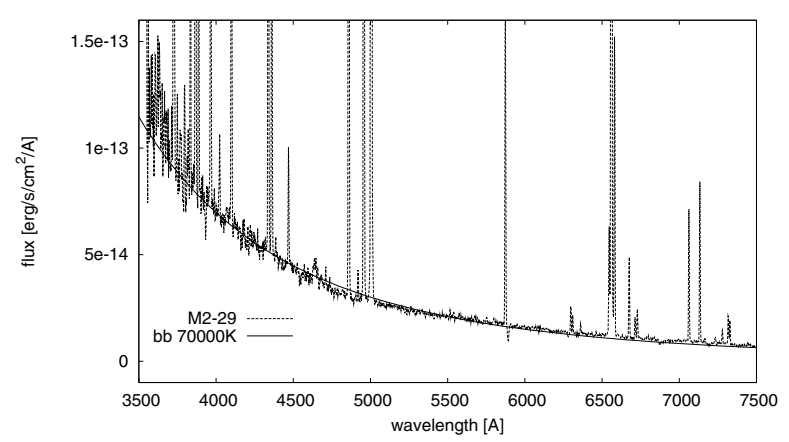

Fig. 4. Dereddened SAAO spectrum of M2-29 along with the blackbody $70 \mathrm{kK}$ model.

show a barely resolved elongated structure superposed on a stellar PSF in the center of the nebula. The sensitivity of the system at $5007 \AA$ varies between the three epochs, which causes systematic offsets in the determination of the stellar brightness. The measured stellar magnitude is very sensitive to the nebular contribution, especially during minimum when the bulk of the measured $V$ band flux comes from the nebular emission. To remove the nebular contribution and obtain absolute photometry, we smoothed the HST [O III] $5007 \AA$ image down to the resolution of the reference image and subtracted it from the reference frame. A scaling factor was chosen such that, after subtraction, a pure stellar PSF was left.

The contribution of the emission line to the OGLE $V$-band photometry was checked with the help of a 1.9-m SAAO telescope low-dispersion optical spectrum obtained on June 16, 2005. The low-resolution ( $5 \AA$ ) spectrum covers the wavelength range 3500-7500 A.

MACHO data is available for M 2-29 for 7 seasons, starting from 1993 (Fig. 3). The blue MACHO filter runs from $\sim 4500$ to $6300 \AA$, including the [O III] 4959/5007 $\AA$ line. The red filter ( 6300-7600 $\AA$ ) includes $\mathrm{H} \alpha$ (Alcock et al. 1997). The MACHO photometry is not calibrated to absolute units. To improve the $\mathrm{S} / \mathrm{N}$, the data points were averaged for each 40 days.

The SAAO spectrum (Fig. 4), similar to the spectra published by Peña \& Torres-Peimbert (1991), shows a nearfeatureless stellar continuum. The He II $4541 \AA$ absorption line (reported by Peña et al. 1992) is confirmed in our spectrum. We dereddened the spectrum of M 2-29 using a standard extinction 
law, with $\mathrm{c}_{\beta}$ of 1.18 , corresponding to $E(B-V)=0.63$, and $A_{V}=1.9$. There is no indication for a binary companion in the spectrum. Our photo-ionization model of the nebula indicates a stellar temperature $T_{\text {eff }} \approx 70 \mathrm{kK}, \log \left(L / L_{\odot}\right)=3.4$ and an ionized mass $M_{\mathrm{i}} \sim 0.48 M_{\odot}$, for a distance of $8 \mathrm{kpc}$. The radius of the central star is about $R_{\star}=2 \times 10^{10} \mathrm{~cm}$.

\section{Discussion}

\subsection{The lightcurve}

Between the beginning of June (JD 2449512) and September 1994 (JD 2449610), for $\sim 100$ days, the I-band magnitude dropped from 14.9 to $16.0 \mathrm{mag}$ (Fig. 2). The drop after JD 2449512 was approximately linear on the magnitude scale, showing tiny variations (Fig. 5) superposed on the linear trend. The final part of the decline was not covered by OGLE. If the drop of the brightness did continue to be linear, the star would have reached the minimum approximately at JD 2449620 , with the full decline taking about 110 days. For the next 210 days, it remained at the minimum level of 16.0 mag. The MACHO data indicate that the minimum remained flat even after the OGLE observations ceased, and the recovery took place somewhere between JD 2450380 and 2450507 and lasted less than 130 days (Fig. 3). The whole event took $\approx 1000$ days.

Out of the minimum, a gradual, linear brightening is seen between 1998 and 2007, by $\Delta I=0.2 \mathrm{mag}$. The continuum was brighter before the eclipse event, suggesting a reversal of this trend occurred around the minimum. Superposed on this trend is a secondary minimum, during 2002-2005, with an amplitude of $\Delta I=0.15$. This secondary minimum is not sampled well regarding its onset. Its duration was similar to that of the primary minimum, but it lacked the flat minimum. The two minima are separated by $\sim 9 \mathrm{yr}$, giving a plausible orbital period of $18 \mathrm{yr}$. Confirmation is needed.

The amplitude of the decline was $\Delta I \sim 1.3 \mathrm{mag}$, and $\Delta V \sim$ 1.7-1.8 mag. MACHO data shows a decline of $\sim 1.0 \mathrm{mag}$ in MACHO blue and $\sim 0.9 \mathrm{mag}$ in MACHO red. The star may have shown somewhat redder colors during minimum than during maximum.

\subsection{Disk occultation}

The notable aspects of the eclipse are the long time scale, the slow ingress and egress, and the relatively shallow depth.

An eclipse by a companion star can be ruled out. The decline took about 100 days, and the whole minimum lasted about 1000 days. This implied a size of the occulting body of $\sim 10 \times$ larger than the central star, or $2 \times 10^{11} \mathrm{~cm}\left(\sim 3 R_{\odot}\right)$, comparable to the size of a main-sequence star. However, the relative tangential velocities would be close to $2 \times R_{\star} / 100 d=25 \mathrm{~m} / \mathrm{s}$, corresponding to an orbit of $\sim 10^{6} \mathrm{AU}$. The eclipsing body could be on a very eccentric orbit, or not bounded gravitationally with the central star; however, this does not change the extreme unlikelihood of such an eclipse event.

The event is fitted better with an occultation by a large screen. The central star of a bulge PN is expected to have a mass $M_{\mathrm{f}} \approx 0.6 M_{\odot}$. Assuming a secondary component with similar mass, the orbital separation for an 18-yr period is $\sim 7 \mathrm{AU}$. The eclipse lasted for about $15 \%$ of the adopted orbital period: this yields a radius of the occulting body of $R \sim 1-5 \mathrm{AU}$, depending on mass ratio and where the occultation occurred on the orbit.

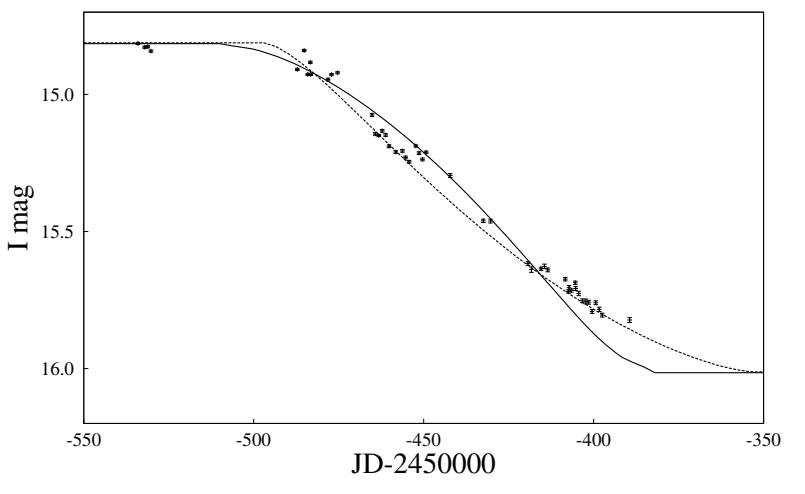

Fig. 5. I light curve of M2-29 from OGLE during the decline. Two models are shown for an eclipse by the object by a sharp-edged screen. Dashed line: a total eclipse of the star + constant source of the flux present (or an eclipse by a semi-transparent cloud). Solid line: partial eclipse by a rectangular, opaque body.

We propose a model where the occultation is caused by a circumbinary (dust) disk. Such disks are now known to be common around post-AGB stars, and especially so around RV Tau stars (van Winckel 2003; Deroo et al. 2007; de Ruyter et al. 2006). Their absence around central stars of PNe has become puzzling. In recent years, the first evidence of such disks have begun to appear, e.g. the discovery of an unexpected compact disk in the Ant nebula (Chesneau et al. 2007), a disk in the helix nebula ( $\mathrm{Su}$ et al. 2007), and the possibly related structure in CK Vul (Hajduk et al. 2007). The NIR colors indicate the presence of $1000 \mathrm{~K}$ dust in M 2-29, as mentioned above, i.e. near the sublimation temperature. The dust sublimation radius is around $10 \mathrm{AU}$, and so the dust is located outside of the orbit of the 18-yr binary.

Among objects with similar events, long-lasting minima are observed in wide binary systems, like symbiotic stars; they are ascribed to dusty clouds. In V Hya such a minimum is observed repeatedly with over a 6000 day period (Knapp et al. 1999). NGC 2346 has shown two long-lasting eclipses by clouds (Kato et al. 2001). Either $\epsilon$ Aur or EE Cep are examples of eclipses by a disk placed nearly edge-on to the observer, around an unseen companion (Carroll et al. 1991; Mikołajewski \& Graczyk 1999). Such eclipses appear to be flat and almost grey.

Many of the obscuration characteristics of M 2-29 are replicated in the "winking" star KH 15D, an eccentric pre-mainsequence binary that is gradually being occulted by an opaque screen (Winn et al. 2006; Herbst et al. 2008). The binary orbit is inclined with respect to the circumbinary disk, and in the current configuration one of the stars experiences a "sunset" and "sunrise" every orbit behind the disk. The lightcurve of KH 15D is very similar to that of M2-29, although the time scales are very different as KH $15 \mathrm{D}$ presents a much more compact system.

The nature of the secondary star in M 2-29 is uncertain. We find no evidence that is has been observed. The depth of the secondary minimum corresponds to a flux only a few times less at $I$ than the PN central star, which is much brighter than any plausible main-sequence star. Such a luminous (red) star would have shown up in the spectra. It is possible that the secondary minimum, and in fact the slow brightening prior, are caused by reflected light from the dusty disk. This would explain the lack of spectral features, the equal length of the secondary minimum (implying a similar orbital radius), and the partial nature of the secondary eclipse, which shows that the secondary emission region is larger than the primary star. 


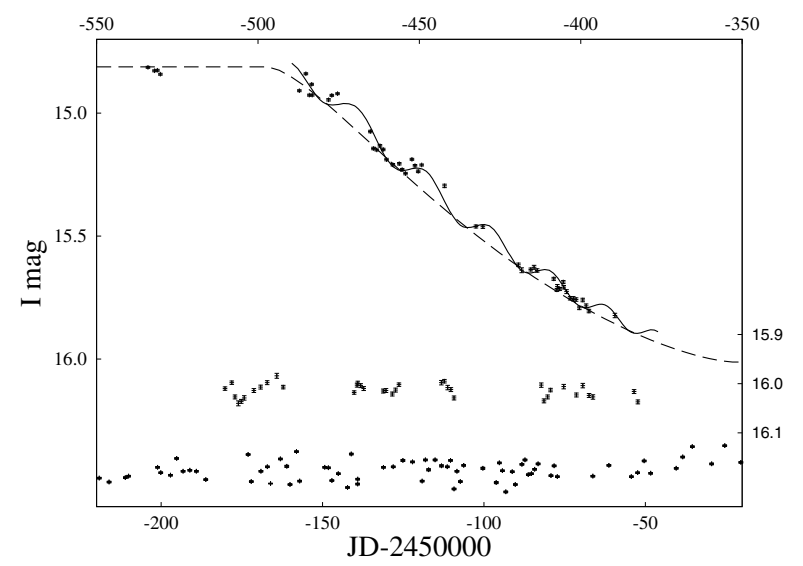

Fig. 6. $I$ light curve of M 2-29 from OGLE during the decline and the minimum. The timescale for points from minimum is shifted to position the data below the decline phase. A sample of variability of the central star out of the minimum is shown at the bottom. The dashed line shows a total eclipse of the central star by a semi-transparent, sharp-edged body with the sharp edge. The solid line includes the $\sim 23$-day and 18-yr orbital motions of the central star.

\subsection{Ionized gas}

In the HST images, the star appears much brighter in $\mathrm{F} 656 \mathrm{~N}$ $(\mathrm{H} \alpha)$ than expected from the F547M continuum image: $V=$ $15.6 \pm 0.1$ versus $m(656 \mathrm{~N}) \approx 12.9$, corresponding to an $\mathrm{H} \alpha$ $E W$ of $\approx 250 \AA$. The stellar magnitude was measured by conversion of the data count from the HST image into the flux and subsequently turned into Johnson magnitudes using Bessel zero points. Torres-Peimbert et al. (1997) also finds that the star is blended with an unresolved, inner emission-line nebula. There may also be an excess in F502N ([O III]), where we estimate $m \sim$ 14.3, which after correction for extinction of $E(B-V)=0.63$ leaves about 1 mag excess. The [ O III] $5007 \AA$ excess can only arise from low-density gas and argues against a stellar wind as the origin of the excess. Instead a circumstellar emission region is indicated.

These data suggest that the light source may be an ionized, inner component to the disk. Such a disk can cause the stellar excess [O III] and $\mathrm{H} \alpha$ emission (Soker 2006). We also tentatively assign the He II absorption seen by Peña et al. (1992) to this disk/component. As the ionized region would be much larger than the stellar radius, this might also help explain the long duration before reaching the minimum.

\subsection{A close binary}

We find evidence of semi-regular photometric variations on a timescale of $\sim 23$ days, which appeared during the decline and the minimum and were present at a level of $\Delta m_{I} \approx$ 0.05-0.1 mag. The amplitude of these variations is a few times greater than the accuracy of the individual data points, which spans 0.004-0.01 mag. This is shown in Fig. 6: the initially regular variations lost phase stability before the star was fully hidden. A similar modulation during the decline was seen for NGC 2346 (Méndez et al. 1982) where the 16-day photometric variations ceased immediately after the minimum. They were attributed to the geometric motion of the primary star due to an undetected, faint companion. As the star moves around the barycenter, the degree of obscuration varies. This gives a photometric modulation on the orbital time scale, where the phase depends on the structure of the occulting material.
Following the case of NGC 2346, we interpret the photometric variability as evidence of a companion to the central star with an orbital period of 23 days. The eclipse may be considered as the result of the orbital motion of the central star behind the obscuring screen. The 18 -yr orbital motion is responsible for the overall shape of the decline, which lasts for over $2 \%$ of the orbital period. The amplitude and period of the photometric modulations of the eclipse can be modeled by an orbital motion of the central star on a $\sim 23$-day orbit (Fig. 6). During each 23-day cycle, there is a phase of nearly constant luminosity, when the velocity vectors cancel each other and the star remains stationary with respect to the obscuring body. Assuming that the outer companion has a mass of $0.5-1 M_{\odot}$ (as expected for bulge star) and that the screen is stationary with respect of the barycenter, this indicates a mass of the close companion of $0.1-0.2 M_{\odot}$.

In M 2-29, the photometric variability re-appeared during the minimum. The phase of the photometric variations is conserved only for 2-3 consecutive periods. This indicates that the optical depth through the eclipsing body is not uniform. Some of the received light may be scattered.

The detection of such a companion to the primary central star is significant. As shown by Peretto et al. (2007), the infall of a distant companion to such a period can provide the energy needed to create an expanding torus. The morphology of M 2-29 clearly shows the existence of such a torus. In contrast, the much shorter-period binaries that have come through a common-envelope evolution show rings, jets, and irregular structures, but no tori or butterfly morphologies (Zijlstra 2007). This would therefore greatly strengthen the case that close binaries have a strong effect on the shaping of planetary nebulae.

Acknowledgements. We acknowledge Prof. Andrzej Udalski and Prof. Michal Szymanski for providing the OGLE data. M.H. thanks Sławek Górny for travel to SAAO and help in retrieving the spectrum. This work was financially supported by MNiSW of Poland through grant No. N 203 02431/3879.

\section{References}

Alcock, C., Allsman, R. A., Alves, D., et al. 1997, ApJ, 486, 697 Carroll, S. M., Guinan, E. F., McCook, G. P., \& Donahue, R. A. 1991, ApJ, 367, 278

Chesneau, O., Lykou, F., Balick, B., et al. 2007, A\&A, 473, L29 De Marco, O., Hillwig, T. C., \& Smith, A. J. 2008, AJ, accepted Deroo, P., Acke, B., Verhoelst, T., et al. 2007, A\&A, 474, L45 de Ruyter, S., van Winckel, H., Maas, T., et al. 2006, A\&A, 448, 641 Gesicki, K., \& Zijlstra, A. A. 2007, A\&A, 467, L29

Hajduk, M., Zijlstra, A. A., van Hoof, P. A. M., et al. 2007, MNRAS, 378, 1298 Herbst, W., Hamilton, C. M., Leduc, K., et al. 2008, Nature, 452, 194 Kato, T., Nogami, D., \& Baba, H. 2001, PASJ, 53, 901

Knapp, G. R., Dobrovolsky, S. I., Ivezić, Z., et al. 1999, A\&A, 351, 97 Livio, M., \& Soker, N. 1988, ApJ, 329, 764

Méndez, R. H., Gathier, R., \& Niemela, V. S. 1982, A\&A, 116, L5

Mikołajewski, M., \& Graczyk, D. 1999, MNRAS, 303, 521

Miszalski, B., Acker, A., Moffat, A. F. J., Parker, Q. A., \& Udalski, A. 2008, A\&A, 488, L79

Peña, M., Torres-Peimbert, S., \& Ruiz, M. T. 1991, PASP, 103, 865

Peña, M., Torres-Peimbert, S., \& Ruiz, M. T. 1992, A\&A, 265, 757

Peretto, N., Fuller, G., Zijlstra, A., \& Patel, N. 2007, A\&A, 473, 207 Soker, N. 2006, ApJ, 640, 966

Su, K. Y. L., Chu, Y. H., Rieke, G. H., et al. 2007, ApJ, 657, L41

Torres-Peimbert, S., Dufour, R. J., Peimbert, M., \& Peña, M. 1997, in IAU Symp. 180, ed. H. J. Habing, \& H. J. G. L. M. Lamers (Dordrecht: Kluwer), 126 Udalski, A., Szymanski, M., Kubiak, M., et al. 2002, AcA, 52, 217 van Winckel, H. 2003, ARA\&A, 41, 391

Wareing, C. J., Zijlstra, A. A., \& O’Brien, T. J. 2007, MNRAS, 382, 1233

Whitelock, P. A. 1985, MNRAS, 213, 59

Winn, J. N., Hamilton, C. M., Herbst, W. J., et al. 2006, ApJ, 644, 510

Zebrun, K. 1998, AcA, 48, 289

Zijlstra, A. A. 2007, Baltic Astron., 16, 79 